\title{
Scaling of the glassy dynamics of soft repulsive particles: a mode-coupling approach
}

\author{
Ludovic Berthier, ${ }^{1}$ Elijah Flenner, ${ }^{2}$ Hugo Jacquin, ${ }^{3}$ and Grzegorz Szamel ${ }^{1,2}$ \\ ${ }^{1}$ Laboratoire des Colloïdes, Verres et Nanomatériaux, \\ UMR CNRS 5587, Université Montpellier 2, 34095 Montpellier, France \\ ${ }^{2}$ Department of Chemistry, Colorado State University, Fort Collins, CO 80523 \\ ${ }^{3}$ Laboratoire Matière et Systèmes Complexes, UMR CNRS 705\%, Université Paris Diderot - Paris 7 , \\ 10 rue Alice Domon et Léonie Duquet, 75205 Paris cedex 13, France
}

(Dated: November 8, 2018)

\begin{abstract}
We combine the hyper-netted chain approximation of liquid state theory with the mode-coupling theory of the glass transition to analyze the structure and dynamics of soft spheres interacting via harmonic repulsion. We determine the locus of the fluid-glass dynamic transition in a temperature volume fraction phase diagram. The zero-temperature (hard sphere) glass transition influences the dynamics at finite temperatures in its vicinity. This directly implies a form of dynamic scaling for both the average relaxation time and dynamic susceptibilities quantifying dynamic heterogeneity. We discuss several qualitative disagreements between theory and existing simulations at equilibrium. Our theoretical results are, however, very similar to numerical results for the driven athermal dynamics of repulsive spheres, suggesting that 'mean-field' mode-coupling approaches might be good starting points to describe these nonequilibrium dynamics.
\end{abstract}

PACS numbers: 05.20.Jj, 64.70.qd

\section{INTRODUCTION}

An assembly of hard spherical particles undergoing Brownian motion, if it can avoid crystallization (e.g. due to polydispersity), at a sufficiently high volume fraction undergoes a glass transition to an amorphous solid state [1]. In experiments, structural relaxation of colloidal hard sphere systems stops near volume fraction $\varphi \approx 0.60[2]$. The phenomenology of this so-called colloidal glass transition is strikingly reminiscent of the molecular glass transition observed upon decreasing the temperature in glass-forming liquids. When Brownian motion is negligible, a hard sphere system undergoes instead a jamming transition near $\varphi \approx 0.64$; it acquires rigidity by building a mechanically stable network of contacts between particles [3]. This is most readily observed in granular materials.

While the glass and jamming transitions of hard sphere systems have been widely studied for a long time, the study of dense systems composed of soft repulsive particles is, by comparison, in its infancy. Colloidal particles with tunable softness are now routinely prepared in the laboratory [4, 5, 6, 7] and examples of compressible grains abound [8, 9, 10]. The rheological, structural, or dynamical properties of both types of systems are currently actively studied experimentally by several groups [4, 5, 6, 7, 8, 9, 10]. This justifies current theoretical efforts to understand the behaviour of dense assemblies of soft repulsive particles both at finite temperatures [11, 12, 13, 14], relevant for colloidal systems, and in the zero-temperature limit relevant for granular materials [15, 16, 17, 18, 19].

In this paper, we combine the hyper-netted chain approximation of liquid state theory [20] with modecoupling theory [21] to analyze the equilibrium structure and dynamics of dense systems of soft particles with finite range, harmonic repulsion [15]:

$$
V(r<\sigma)=\epsilon(1-r / \sigma)^{2},
$$

where $\epsilon$ determines the strength of the repulsion, $\sigma$ is the particle diameter, and $r$ the distance between two particles. Particles separated by $r>\sigma$ do not interact, $V(r>\sigma)=0$. The control parameters for this system are therefore the volume fraction $\varphi=\pi \sigma^{3} \rho / 6$, with $\rho$ the number density, and the ratio of the temperature and $\epsilon$. In the following we use reduced units: we give lengths in units of $\sigma$ and temperature in units of $\epsilon$.

The system of harmonic spheres was originally introduced in the context of the zero-temperature jamming transition [15]. More recently, its behaviour was investigated at finite temperature using molecular dynamics computer simulations [11, 12, 13]. To our knowledge, this model system was not studied theoretically in the context of liquid state and mode-coupling theories. However, its physics should be similar to a number of similar models such as Hertzian spheres 22] or the Gaussian core model [23, 24].

In both thermal and athermal contexts various scaling relations were reported for the dynamics of harmonic spheres in the vicinity of the glass or jamming transitions occurring in the hard sphere limit [11, 12, 18, 19]. This type of scaling is usually rationalized by postulating that harmonic spheres in fact behave as an 'effective' hard sphere system with a 'renormalized' volume fraction [11]. However, the scaling formulae are typically established using largely empirical procedures. Our primary goal in this work is to derive this scaling behaviour using a liquid state theoretical approach in order to put it on a firmer basis, at least for systems in thermal equilibrium.

The paper is organized as follows. We present theoretical methods which we use to obtain the structure 
and dynamics of harmonic spheres in Sec. II. We then present results for the equilibrium dynamics in Sec. [II] We describe the phase diagram in Sec. IV In Sec. V we analyze dynamic heterogeneity using three-point dynamic susceptibilities. We conclude the paper in Sec.VI.

\section{THEORETICAL APPROACH}

Our theoretical approach consists of two steps. First, we use the hyper-netted chain (HNC) approximation of liquid state theory to obtain the static structure of the harmonic sphere system. Next, we use the predicted static structure factor as input of the mode-coupling equations to obtain time correlation functions of harmonic spheres and we use these functions to determine the dynamical behaviour. In the next two subsections we briefly describe the two elements of our approach.

\section{A. HNC equations and static behaviour}

The HNC approximation [20] results in a closed equation for the pair correlation $g(r)$ of a fluid. It reads:

$$
g(r)=\exp [-\beta V(r)+g(r)-1-c(r)]
$$

where $\beta=1 / T$ and $c(r)$ is the direct correlation function defined through the Ornstein-Zernike equation:

$$
g(r)-1=c(r)+\rho \int d r^{\prime} c\left(\left|r-r^{\prime}\right|\right)\left[g\left(r^{\prime}\right)-1\right] .
$$

We solve Eq. (2) numerically using an iterative procedure. Since the direct correlation function is smoother than the pair correlation function, the HNC equation is more easily solved in terms of $c(r)$. Given the solution for $c(r)$ after $i-1$ iterations, $c_{i-1}(r)$, we obtain the solution at step $i$ as follows:

$$
\begin{aligned}
& c_{i-1}(r) \stackrel{\mathrm{FT}}{\longrightarrow} \hat{c}_{i-1}(q) \stackrel{\mathrm{OZ}}{\longrightarrow} \hat{g}_{i-1}(q) \stackrel{\mathrm{FT}^{-1}}{\longrightarrow} g_{i-1}(r) \\
& \stackrel{\mathrm{HNC}}{\longrightarrow} c(r) \rightarrow c_{i}(r)=\alpha c(r)+(1-\alpha) c_{i-1}(r),
\end{aligned}
$$

where the first and third steps are Fourier transforms performed using fast Fourier transforms, the second one uses the Ornstein-Zernike relation (3), the fourth one uses the HNC closure relation (2), and the last step involves combining the new direct correlation function $c(r)$ with $c_{i-1}(r)$ using a mixing parameter $\alpha$. Convergence is achieved when the difference between $c_{i}(r)$ and $c_{i-1}(r)$ in Eq. (4) becomes smaller than some prescribed precision.

Since we want to access very low temperatures where the pair potential in Eq. (1) becomes equivalent to a hard sphere potential, some attention must be paid to the discretization scheme we use. Additionally, the fluid develops medium-range structure when density increases and/or temperature decreases, so that the cut-off in real space must be large enough. We used a real space cutoff $L=32$, and discretized the interval $[0, L]$ using $2^{n}$

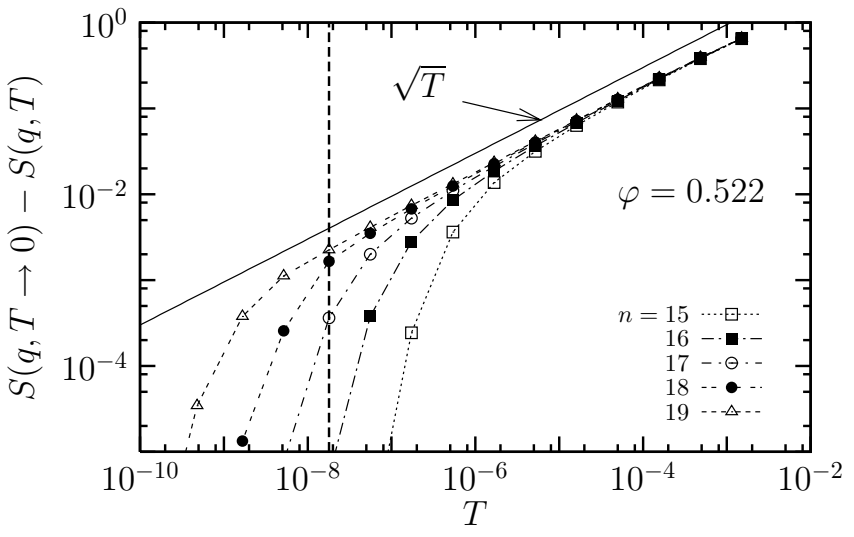

FIG. 1: Convergence of the numerical solution of the HNC structure factor to its $T=0$ hard sphere limit for $q$ near the first diffraction peak at $\varphi=0.522$. For a given number $2^{n}$ of discretized values of $r$ used to solve Eq. (2), there exists a temperature above which the $\sqrt{T}$ convergence in Eq. (17) is obeyed. The vertical line shows the lowest value of $T$ used in this work.

points, which is convenient for the fast Fourier transform algorithm. To properly represent the low temperature behaviour, discretization must be accurate enough that the factor

$$
Y(r, T) \equiv \exp \left[-\beta(1-r)^{2}\right]
$$

is correctly described even at very low $T$. We note that, for a given discretization, there necessarily exists a temperature, $T_{n}$, below which $Y(r, T)$ is not welldescribed. It is easy to see that this temperature scales as $T_{n} \sim 2^{-2 n}$.

This behaviour is ilustrated in Fig. 1 which shows how the structure factor $S(q, T)$ of harmonic spheres at $\varphi=0.522$ reaches its $T \rightarrow 0$ value for increasingly finer discretizations (data are shown for $q$ near the first diffraction peak). To get accurate results down to $T=10^{-8}$, as shown below, we need to use $n>18$. The value $n=19$ is used throughout this paper. Additionally, since we need structure factors for temperatures and densities in very narrow ranges near the dynamic singularities, we had to pay close attention to the accuracy of the numerical solution of the HNC equation in order to resolve very close state points.

In Fig. 1 we also show that difference between the finite temperature $S(q, T)$ and its hard sphere $(T=0)$ limit scales as $\sqrt{T}$. This behaviour can be derived as follows. Since $g(r<1, T=0)=0$ for hard spheres we can decompose the difference $S(q, T)-S(q, 0)$ as follows

$$
\begin{aligned}
S(q, T)-S(q, 0) & =4 \pi \rho \int_{0}^{1} d r r^{2} \frac{\sin q r}{q r} g(r, T) \\
& +4 \pi \rho \int_{1}^{\infty} d r r^{2} \frac{\sin q r}{q r}[g(r, T)-g(r, 0)] .
\end{aligned}
$$

Using the fact that in the low temperature $T \rightarrow 0$ limit $Y(r, T)$ becomes a narrow peak located at $r=1$, it is 
possible to approximate the first term in this expression by

$$
2 \pi^{3 / 2} \rho y(r=1, T=0) \frac{\sin q}{q} \sqrt{T} .
$$

where $y(r, T)=g(r, T) / Y(r, T)$ is the cavity function. Since both $g(r, T)-g(r, 0)$ and $S(q, T)-S(q, 0)$ are of the same order, we conclude that

$$
S(q, T)-S(q, 0) \sim \sqrt{T},
$$

and this scaling is satisfied, see Fig. 1, The $\sqrt{T}$ temperature dependence of the difference $S(q, T)-S(q, 0)$ will play a crucial role in the next section, Sec. III. We will use it to motivate the dynamic scaling in the vicinity of the hard-sphere transition, within the mode-coupling approximation.

In a recent molecular dynamics investigation [11], the temperature dependence of the energy density, $e(T)$, was used to relate soft spheres to hard particles. We can use a similar reasoning to predict the low temperature behaviour of $e(T)$. The energy density can be expressed in terms of the pair correlation function

$$
e(T)=2 \pi \rho \int_{0}^{1} d r r^{2} V(r) g(r, T) .
$$

The convergence of $e(T)$ to its $T=0$ value $e(T=0)=0$ can be estimated by making explicit the $Y(r, T)$ factor in $g(r, T)$ and using the cavity function. In this way we obtain

$$
e(T) \approx \frac{\pi^{3 / 2}}{2} \rho y(r=1, T=0) T^{3 / 2} .
$$

Molecular dynamics investigation [11] reported a power law behaviour of the energy density, $e(T) \sim T^{\mu}$, with exponent $\mu$ crossing over from $\mu=3 / 2$ at low volume fraction to $\mu \approx 1.3$ at larger volume fraction. This indicates that the low temperature scaling regime (9) is not accessible at large densities in molecular dynamics simulations.

\section{B. MCT analysis and dynamic behaviour}

The mode-coupling theory (MCT) 21] was originally derived to describe the dynamics of Newtonian systems [25]. An analogous theory was later derived for Brownian systems [26]. Here we briefly present the latter version of MCT.

The starting point of the theory is an exact equation for the time derivative of the intermediate scattering function $F(q ; t)$ in terms of the so-called irreducible memory function,

$\partial_{t} F(q ; t)=-\frac{D_{0} q^{2}}{S(q)} F(q ; t)-\int_{0}^{t} d t^{\prime} M^{\mathrm{irr}}\left(q ; t-t^{\prime}\right) \partial_{t^{\prime}} F\left(q ; t^{\prime}\right)$.
Here $D_{0}$ is the diffusion coefficient of an isolated Brownian particle. Irreducible memory function $M^{\text {irr }}(q ; t)$ can be expressed in terms of a time-dependent four-point density correlation function evolving with the so-called irreducible dynamics. The main approximation of MCT consists in factorizing this four-point function. In this way this somewhat mysterious quantity is reduced to a product of two intermediate scattering functions. After an additional technical approximation (which was independently shown to be quite innocuous) one arrives with the following expression for the irreducible memory function

$$
\begin{aligned}
& M^{\operatorname{irr}}(q ; t)=\frac{\rho D_{0}}{2 q^{2}} \int \frac{d \mathbf{q}_{1}}{(2 \pi)^{3}}\left[\mathbf{q} \cdot \mathbf{q}_{1} \hat{c}\left(q_{1}\right)\right. \\
& \left.+\mathbf{q} \cdot\left(\mathbf{q}-\mathbf{q}_{1}\right) \hat{c}\left(\left|\mathbf{q}-\mathbf{q}_{1}\right|\right)\right]^{2} F\left(q_{1} ; t\right) F\left(\left|\mathbf{q}-\mathbf{q}_{1}\right| ; t\right) .
\end{aligned}
$$

Equations (10-11) allow us to evaluate the time dependence of the intermediate scattering function. The only input required is the static structure factor $S(q)$. It enters into Eqs. (10 11) (note that $\hat{c}(q)=(1-1 / S(q)) / \rho$, from Eq. (31) and it also provides the initial condition for the intermediate scattering function, $F(q ; t=0)=S(q)$. It is easy to see that the natural time unit for our system of harmonic spheres is $\sigma^{2} / D_{0}$. In the following all times are given in terms of this unit.

Numerical solution of Eqs. 10 111) is somewhat complicated because one needs to describe evolution of the intermediate scattering function on very widely separated time scales (see Fig. 22). The commonly used algorithm was first described in Ref. [27]; here we use the implementation described in considerable detail in Ref. [28]. Briefly, the basic steps to the algorithm are as follows. The integro-differential equation is discretized and solved for $2 N_{s}$ steps with a finite time step of $\delta t$ using any suitable numerical algorithm. After $2 N_{s}$ steps are complete, the time step is doubled and the results from the initial $2 N_{s}$ steps are mapped into a new equally spaced set of $N_{s}$ values for the quantities needed to continue the numerical algorithm. This mapping includes the integrals as well as the intermediate scattering functions. Then the numerical algorithm is restarted with the new time step and continued for another $N_{s}$ time steps and the mapping is performed again. This procedure is continued until a convergence condition is satisfied. In the present work we used 300 equally spaced wave-vectors with spacing $\delta \approx 1.96$, the first wave-vector at $k_{0}=\delta / 2$ and the largest wavevector at $k_{\max } \approx 58.81$.

In Fig. 2 we show the prediction of the mode-coupling theory for the time dependence of the normalized intermediate scattering function $F(q ; t) / S(q)$ for harmonic spheres at $\varphi=0.5235$. With decreasing temperature the relaxation becomes progressively slower. One should notice that an intermediate time plateau is developing which is the manifestation of the cage effect: with decreasing temperature particles are trapped longer and longer within their first solvation shells and the final $(\alpha)$ relaxation shifts to longer and longer times. MCT predicts that at this volume fraction at temperature 


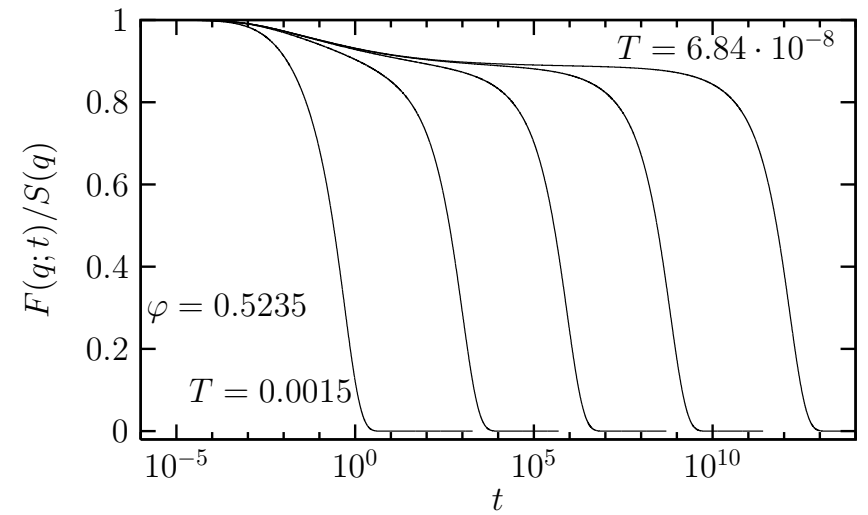

FIG. 2: Time dependence of the normalized intermediate scattering function $F(q ; t) / S(q)$ as predicted by the modecoupling theory for harmonic spheres at $\varphi=0.5235$, for wavevector corresponding the first peak in the static structure factor, $q=q_{\max } \approx 7.17$. The static structure factor used as input in mode-coupling equations was obtained from the hyper-netted chain approximation. The lines correspond to the following temperatures (from left to right): 0.0015 , $1.61 \times 10^{-5}, 5.39 \times 10^{-7}, 1.00 \times 10^{-7}$, and $6.84 \times 10^{-8}$.

$T_{c}(\varphi=0.5235) \approx 6.594 \times 10^{-8}$ the plateau extends to infinite times and the system undergoes an ergodicity breaking transition which is commonly referred to as the glass transition.

In this work we are primarily focused on the temperature and volume fraction dependence of the $\alpha$ relaxation time which we define in the standard way, $F\left(q_{\max } ; \tau_{\alpha}\right)=$ $e^{-1}$, where $q_{\max }$ is the position of the first peak in the static structure factor. MCT makes a number of detailed predictions for various aspects of the time dependence of intermediate scattering functions (including power law approach to and departure from the intermediate time plateau, and the wavevector dependence of the relaxation time). The investigation of the temperature and volume fraction dependence of these properties are left for future work.

The hard sphere glass transition within MCT is usually investigated using as the input the static structure factor obtained from the Percus-Yevick (PY) approximation. For the discretization used in this work MCT combined with the PY structure factor predicts the glass transition at $\varphi_{c}^{\mathrm{PY}} \approx 0.5159$. Moreover, MCT predicts that upon approaching $\varphi_{c}^{\mathrm{PY}}$ the $\alpha$ relaxation time diverges algebraically, $\tau_{\alpha} \sim\left(\varphi_{c}^{\mathrm{PY}}-\varphi\right)^{-\gamma^{\mathrm{HS}, \mathrm{PY}}}$, with the exponent $\gamma^{\mathrm{HS}, \mathrm{PY}} \approx 2.59$.

In this work we use the hyper-netted chain approximation for the static structure factor because we are mostly interested in effects of finite temperature. However, we anticipate that the low temperature results will be influenced by the behavior of the hard sphere system. Therefore, we also solved MCT equations in the $T \rightarrow 0$ limit using as input the structure factor predicted by the hyper-netted chain approximation in this limit. For the discretization used in this work MCT combined with the HNC structure factor predicts the glass transition at $\varphi_{c} \approx 0.52315$. Moreover, according to MCT upon approaching this critical volume fraction the $\alpha$ relaxation time diverges algebraically,

$$
\tau_{\alpha}^{\mathrm{HS}}(\varphi) \approx \frac{\tau_{0}}{\left(\varphi_{c}-\varphi\right)^{\gamma^{\mathrm{HS}}}}
$$

with the exponent $\gamma^{\mathrm{HS}} \approx 3.26$.

It is well known that in real colloidal systems the glass transition predicted by mode-coupling theory is avoided. Typically, as shown in recent contributions [2, 12, 29], one can find an intermediate range of volume fractions in which the volume fraction dependence of the $\alpha$ relaxation time can be fitted to a power law with the exponent close to that predicted by MCT (one should note that when this procedure is used the critical volume fraction is one of the fitting parameters; typical values obtained from fits are about $10 \%$ different from MCT predictions). The result is that MCT-predicted power law divergence of the $\alpha$ relaxation time describes well the experimental and simulational data over approximately 3 decades of $\tau_{\alpha}$. Recent experimental and simulational results reported in Refs. 2, 12, 29] suggest that upon increasing volume fraction further this approximate power law is followed by an 'activated' regime according to which the $\alpha$ relaxation time has an essential singularity divergence at a higher volume fraction.

\section{DYNAMIC SCALING AT EQUILIBRIUM}

The tools described in the previous section allow us to obtain, for any given state point $(\varphi, T)$, the relaxation time $\tau_{\alpha}(\varphi, T)$ of the harmonic sphere system within the MCT approximation. We will report results for a broad range of volume fractions, $\varphi \in[0.51,0.90]$, and temperatures, $T \in\left[10^{-8}, 10^{-2}\right]$.

In Fig. 3 we show the evolution of $\tau_{\alpha}(\varphi, T)$ in the vicinity of the hard sphere glass transition occurring at $T=0$ and $\varphi_{c} \approx 0.52315$. The behaviour observed at finite temperature is easily explained. When $\varphi<\varphi_{c}$, the relaxation time increases when $T$ decreases, but it saturates at low temperature to its hard sphere value which is finite at these densities. When increasing the volume fraction closer to $\varphi_{c}$, this hard sphere value becomes larger, and the low temperature limit is reached at a lower temperature. For $\varphi>\varphi_{c}$ the system is a hard sphere glass in the $T \rightarrow 0$ limit and so $\tau_{\alpha}(\varphi, T \rightarrow 0)=\infty$. It is clear, however, that the system hits a finite temperature singularity at a critical temperature, $T_{c}(\varphi)$ which increases continuously from $T_{c}\left(\varphi=\varphi_{c}\right)=0$ when $\varphi$ increases.

At this stage of the description, these data resemble the ones found in numerical simulations [11, 12]. In Refs. [11, 12] a scaling analysis of the relaxation time was performed assuming that harmonic spheres at low temperature resemble an 'effective' fluid of hard spheres. Physically, this means that the softness of the potential 


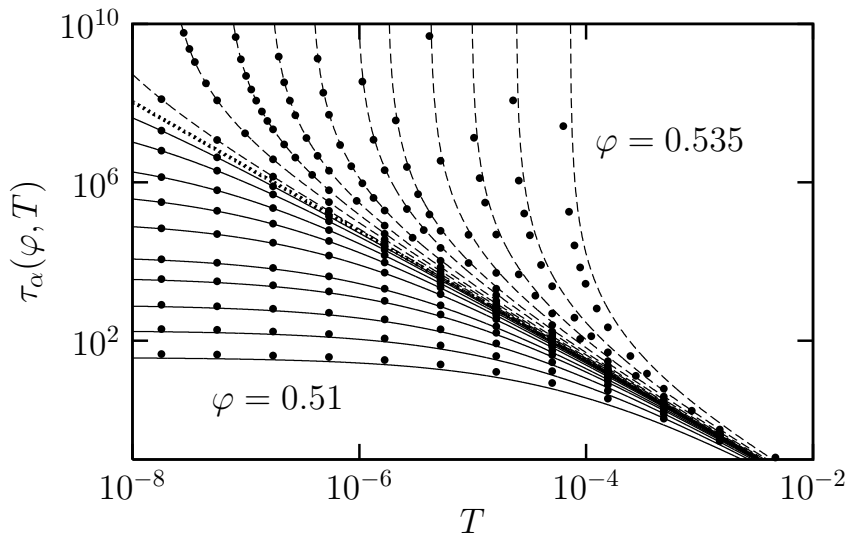

FIG. 3: Relaxation time of harmonic spheres as a function of temperature for various volume fractions in the vicinity of the hard sphere glass transition. The lines are the analytical formula (14) using the scaling functions in Eq. (20); full lines correspond to $\varphi<\varphi_{c}$, dashed lines to $\varphi>\varphi_{c}$. The power law in Eq. (17) for $\varphi=\varphi_{c} \approx 0.52315$ is shown with a dotted line. Corrections to scaling are seen for $\varphi \leq 0.515$ and $\varphi \geq 0.53$. Volume fractions are: (i) $0.51,0.515,0.518$, $0.52,0.521,0.522,0.5225,0.5228,0.523,0.5231$ (ii) 0.5232 , $0.52334,0.5235,0.5237,0.524,0.5245,0.525,0.526,0.5275$, $0.53,0.535$.

allows small overlaps between particles at low temperatures, so that the effective radius of the particles is reduced by thermal fluctuations. While the temperature dependence of the energy density was used to estimate the average overlap, and in turn, the effective hard sphere diameter in Ref. [11], in the context of the mode-coupling approach a different route should be used to map soft to hard particles.

Within MCT, at a fixed number density the dynamics of the system is uniquely controlled by the evolution of the static structure factor. This suggests that the most efficient way to map soft to hard particles is by matching the structure factor of harmonic spheres at $(\varphi, T)$ to the one of a hard sphere system at an effective volume fraction $\varphi_{\text {eff }}=\varphi_{\text {eff }}(\varphi, T)$. Combining the low temperature behaviour of $S(q, T)$ shown in Eq. (7) with the fact that $S(q)$ is smooth function of the volume fraction we easily find that

$$
\varphi_{\mathrm{eff}}(\varphi, T) \approx \varphi-c \sqrt{T}
$$

where $c$ is a positive prefactor with subleading dependencies on temperature and volume fraction.

The discussion in the two previous paragraphs leads to the following form of dynamic scaling that should be obeyed by the relaxation time of the harmonic sphere system:

$$
\tau_{\alpha}(\varphi, T) \approx \frac{\tau_{0}}{\left|\varphi_{c}-\varphi\right|^{\mathrm{HS}}} f_{ \pm}\left(\frac{\left|\varphi_{c}-\varphi\right|}{\sqrt{T}}\right)
$$

where the scaling functions $f_{ \pm}(x)$ respectively refer to volume fractions above and below $\varphi_{c}$. To be consistent

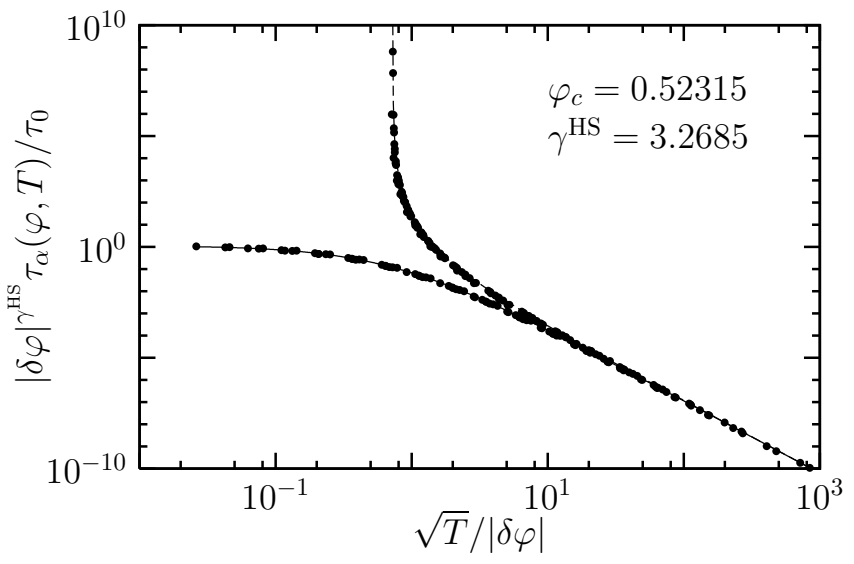

FIG. 4: Collapse of the data shown in Fig. 3 using the scaled variables suggested by Eq. (14) for all data in the range $0.515<\varphi<0.53$. This data collapse involves no free parameter. The lines through the points represent the empirical scaling functions $f_{ \pm}(x)$ in Eq. (20).

with the qualitative behaviour described above, the scaling functions $f_{ \pm}(x)$ must have the following limiting behaviors: to recover the hard sphere plateau at low $T$ below $\varphi_{c}$ we need to have

$$
f_{-}(x \rightarrow \infty) \sim \text { const }
$$

whereas to have a well-behaved $\tau_{\alpha}(\varphi, T>0)$ across $\varphi_{c}$ we have to require

$$
f_{-}(x \rightarrow 0) \sim f_{+}(x \rightarrow 0) \sim x^{\gamma^{\mathrm{HS}}} .
$$

The latter limiting behavior implies that

$$
\tau_{\alpha}\left(\varphi=\varphi_{c}, T\right) \sim\left(\frac{1}{T}\right)^{\gamma^{\mathrm{HS}} / 2}
$$

Finally, a finite temperature algebraic singularity is obtained for $\varphi>\varphi_{c}$ if there exists some $x_{\star}$ such that

$$
f_{+}\left(x \rightarrow x_{\star}^{-}\right) \sim\left(x_{\star}-x\right)^{-\gamma^{\mathrm{HS}}} .
$$

We have found excellent agreement of the MCT predictions with the scaling form in Eq. (14), which suggests that the relaxation times at various $\varphi$ and $T$ can all be collapsed along two branches by plotting the rescaled time, $|\delta \varphi|^{\gamma^{\mathrm{HS}}} \tau_{\alpha}(\varphi, T)$, as a function of the rescaled distance to the critical point, $|\delta \varphi| / \sqrt{T}$, where $\delta \varphi \equiv \varphi_{c}-\varphi$. The data collapse is presented in Fig. 4. It works remarkably well for the range of volume fraction $0.515<\varphi<$ 0.53. It should be noted that no free parameter is involved in this data collapse, which is uniquely controlled by the $T=0$ hard sphere results from Eq. (12), and by using the appropriate relation between temperature and density from Eq. (13). In Refs. [11, 12], a similar data collapse was used in the opposite direction to infer the hard sphere behaviour from the finite temperature dynamics of the harmonic sphere system, a philosophy 
which is clearly supported by the results presented in this section.

The next step is to take Eq. (13) more literally and to combine it with the results for the dynamics of the hard sphere system discussed in Sec. IIB to make the following ansatz

$$
\tau_{\alpha}(\varphi, T) \approx \tau_{\alpha}^{\mathrm{HS}}\left[\varphi_{\mathrm{eff}}(\varphi, T)\right]
$$

Eq. (19) leads to the following scaling functions:

$$
f_{ \pm}(x)=\left(\frac{1}{x} \mp a\right)^{-\gamma^{\mathrm{HS}}}
$$

with $a \approx 0.72$ being the only adjustable numerical factor (note that $a$ is related to $c$ in Eq. (13)) since the values $\gamma^{\mathrm{HS}}=3.26$ and $\varphi_{c}=0.52315$ are directly taken from hard sphere results, while the scaling variable $x=$ $|\delta \varphi| / \sqrt{T}$ was derived in Sec. ПA Clearly, these scaling functions are fully compatible with the constraints described in Eqs. (15, 16, 18). The scaling functions (20) are shown as lines in Figs. 3 and 4 .

We have noted several times the similarity between the present theoretical results and the numerical results and analysis in Refs. [11, 12]. In the simulations, a scaling analogous to the one in Fig. 4 was presented for the behaviour of $\log \tau_{\alpha}(\varphi, T)$ instead of $\tau_{\alpha}(\varphi, T)$ here. This implies that the scaling behaviour predicted by MCT is in fact in strong quantitative disagreement with numerical results. This should not come as a surprise since MCT is not able to describe the thermally activated relaxation which takes place in real glass-formers. Thus, MCT predicts algebraic divergences which are never observed in simulations and experiments, and are replaced by stronger, generically exponential, divergences.

It is interesting to note that algebraic scaling behaviours and divergences seem to be well obeyed in the case of non-equilibrium driven athermal dynamics studied in Refs. [15, 18, 19]. This is again not surprising since in these dynamics the system simply relaxes to the nearest energy minimum without being able to cross energy barrier using thermal activation [30]. This suggests that mode-coupling approaches and 'mean-field' models (see, e.g. Ref. [31]) might well be excellent starting points to tackle the athermal driven dynamics of soft repulsive spheres.

\section{PHASE DIAGRAM}

In this section, we move from scaling properties very near $\varphi_{c}$ and give a broader perspective on the behaviour of the system in the $(\varphi, T)$ phase diagram. The scaling results presented above suggest that the system is ergodic at all temperatures when $\varphi \leq \varphi_{c}$. For $\varphi \gtrsim \varphi_{c}$, Eq. (20) predicts that the relaxation time diverges at $\tau_{\alpha} \sim(T-$ $\left.T_{c}(\varphi)\right)^{-\gamma^{\mathrm{HS}}}$ with a critical temperature which vanishes continuously at $\varphi_{c}$ as:

$$
T_{c}\left(\varphi \geq \varphi_{c}\right) \sim\left(\varphi-\varphi_{c}\right)^{2}
$$

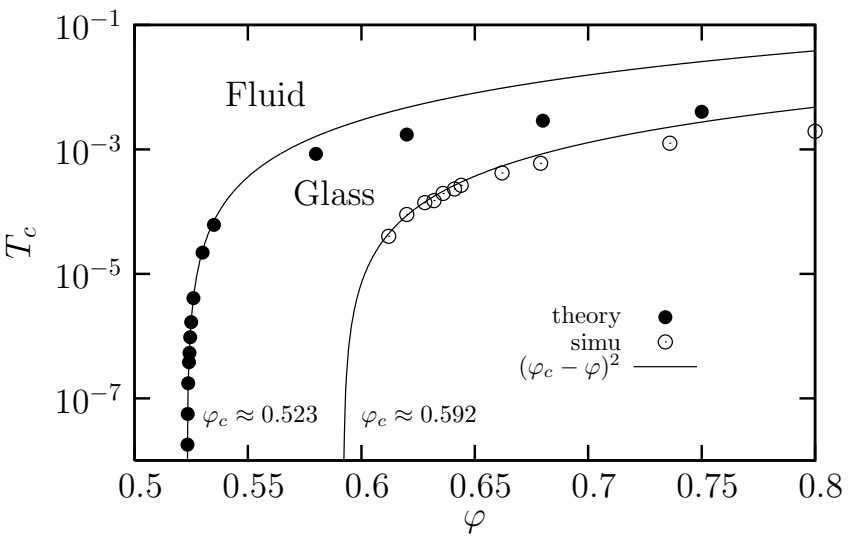

FIG. 5: Phase diagram obtained from the MCT analysis. Filled circles are transition temperatures obtained in this work, which follow the scaling behaviour in Eq. (21) in the vicinity of $\varphi_{c}$, as shown by the full line. Open symbols are the mode-coupling critical temperatures obtained in Ref. [12].

Since the scaling behaviour in Eq. (14) only holds up to $\varphi \approx 0.53$ we have fitted the temperature dependence of the relaxation time at larger volume fraction to the usual power law divergence found within MCT,

$$
\tau_{\alpha}(\varphi, T) \sim\left(T-T_{c}(\varphi)\right)^{-\gamma(\varphi)},
$$

using $T_{c}(\varphi)$ and $\gamma(\varphi)$ as fitting parameters.

In Fig. 5 we show the evolution of the resulting $T_{c}(\varphi)$ which thus delimits the fluid and glass phases in the theoretical phase diagram of the system. These data confirm that the scaling behaviour in Eq. (21) of the critical temperature is only obeyed in the vicinity of $\varphi_{c}$, and clear deviations are seen at larger volume fractions where the scaling prediction overestimates $T_{c}$ by quite a large amount.

We also find that increasing the volume fraction affects the value of the critical exponent $\gamma$. While $\gamma\left(\varphi \approx \varphi_{c}\right)=$ $\gamma^{\mathrm{HS}} \approx 3.26$, we find that $\gamma$ decreases rapidly when $\varphi$ increases: $\gamma(0.58) \approx 2.92, \gamma(0.62) \approx 2.71, \gamma(0.68) \approx 2.50$, $\gamma(0.75) \approx 2.41$, and $\gamma(0.90) \approx 2.37$ This is a clear indication that when moving away from $\varphi_{c}$ the system also leaves the universality class of the hard sphere transition. This means that it becomes impossible to describe the soft spheres as 'renormalized' hard spheres when $\varphi$ becomes too large.

We confirm this statement in Fig. 6 where we show the evolution of the static structure factor along the critical line $T_{c}(\varphi)$. While nearly perfect collapse of the data is obtained for $\varphi_{c} \leq \varphi \leq 0.53$, small deviations become noticeable for $\varphi \approx 0.535$, and are considerably amplified when $\varphi$ increases further. It is this large difference in the shape of the structure factor at the critical temperature which accounts, within MCT, for the continuous evolution of the critical exponent $\gamma$.

We can again compare these theoretical predictions to the numerical analysis reported in Ref. [12]. Although the mode-coupling algebraic singularity is not observed 


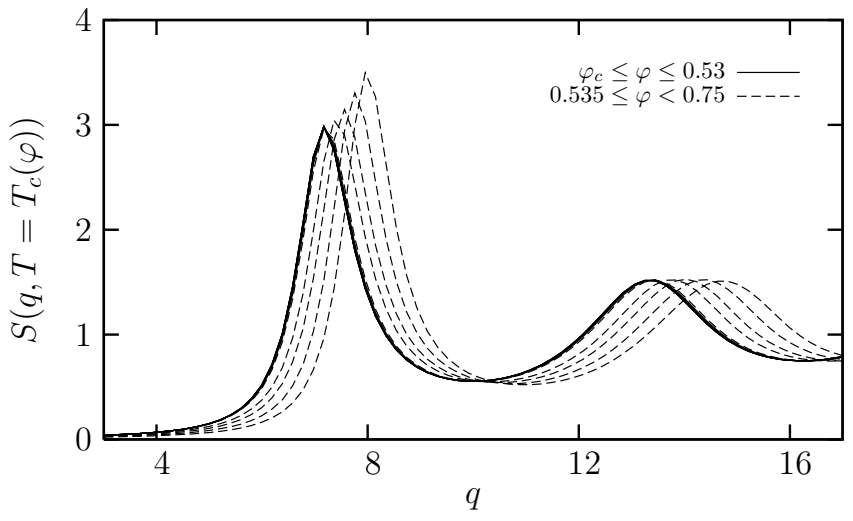

FIG. 6: Evolution of the static structure factor along the MCT critical line $T_{c}(\varphi)$. Full lines show that volume fractions between $\varphi_{c}=0.52315$ and $\varphi=0.53$ collapse on the hard sphere structure factor at $\varphi_{c}$, while deviations appear and increase rapidly at larger $\varphi$ showing that soft spheres are not simply 'renormalized' hard spheres far above $\varphi_{c}$. Volume fractions are: (i) $0.5234,0.5235,0.5237,0.524,0.5245,0.525$, $0.526,0.5275,0.53$. (ii) $0.535,0.58,0.62,0.68,0.75$.

in real liquids, it is usually found that such a power law behaviour is obeyed over a limited time window of approximately 3 decades, which allows a rough determination of the location of the 'avoided' mode-coupling singularity. The outcome of this exercise for the dynamics of harmonic spheres as determined in computer simulations reported in Ref. [12] is shown in Fig. [5 with open symbols. The mode-coupling line determined numerically has qualitatively the same behaviour as the theoretical line. Although the data do not span a very large temperature window, they are indeed compatible with a power law scaling as in Eq. (21) near the hard sphere mode-coupling singularity $\varphi_{c}$, and the critical line becomes smaller than the scaling prediction at larger density. However, the theory is quantitatively inaccurate as it significantly overestimates the critical temperatures at all $\varphi$. Note that for hard spheres MCT combined with HNC structure factor underestimates $\varphi_{c}$ by an amount comparable to that reported for MCT combined with PY structure factor. These discrepancies are well-known features of the mode-coupling approach [21], and MCT descriptions of real data usually imply analysis of scaling behaviour near singularities whose locations must be selfconsistently determined by fitting.

A more surprising disagreement between theory and simulations is the evolution of the critical exponent with density. While theory predicts a substantial decrease of $\gamma$ at large volume fraction, numerical results indicate that $\gamma$ increases instead very rapidly with $\varphi$ above the hard sphere value [12]. It is not clear whether this disagreement stems from an incorrect prediction of the liquid structure by the HNC closure, or from the mode-coupling approach itself.

\section{DYNAMIC HETEROGENEITY}

A newer and lesser known application of MCT is to use it to estimate the strength of dynamic heterogeneity accompanying the glass transition using multi-point dynamic susceptibilities [32]. It is well-known that dynamics near the glass transition is spatially heterogeneous, meaning that different parts of the system relax at different rates, while relaxation is correlated over a lengthscale which increases when the glass transition is approached [33].

A useful tool to quantify the strength of dynamic heterogeneity is the four-point dynamic susceptibility $\chi_{4}(t)$ which is defined from the spontaneous fluctuations of time correlation functions 34,35$]$ :

$$
\chi_{4}(t)=N\left[\left\langle f^{2}(q ; t)\right\rangle-\langle f(q ; t)\rangle^{2}\right],
$$

where $f(q ; t)$ represents the instantaneous value of the intermediate scattering function $F(q ; t)$. Intuitively, $\chi_{4}(t)$ increases if correlations within the system get large, as the number of independently relaxing units within the sample decreases [36]. Formally, $\chi_{4}(t)$ is also the volume integral of a spatial correlator quantifying the extent of correlations between local, spontaneous fluctuations of the dynamics and can thus directly be considered as a proxy for the number of particles that relax in a correlated manner close to the glass transition 37].

We build on the results of Refs. [38, 39, 40] and estimate the spontaneous dynamical fluctuations quantified by $\chi_{4}(t)$ using linear response theory:

$$
\chi_{4}(t) \simeq \frac{T^{2}}{c_{V}}\left(\frac{\partial F(q ; t)}{\partial T}\right)^{2}+S(0, T) \varphi^{2}\left(\frac{\partial F(q ; t)}{\partial \varphi}\right)^{2} .
$$

This relation is known to be an accurate representation of $\chi_{4}(t)$ within the MCT approach [39] and amounts to measuring the response of the averaged dynamics to external fields in the linear regime [38].

The expression in Eq. (24) is highly convenient in the present context as we can directly obtain analytical results for the scaling behaviour of $\chi_{4}(t)$ in the vicinity of $\varphi_{c}$ using results from the previous sections. Since we are interested in the scaling properties of the dynamic susceptibility, we make two further approximations to obtain an analytical form. We first use the fact that the time decay of the intermediate scattering function obeys time temperature superposition, $F(q ; t) \simeq \mathcal{F}\left(t / \tau_{\alpha}\right)$. Thus we have (with $x=T, \varphi$ ):

$$
\frac{\partial F(q ; t)}{\partial x}=-\frac{t}{\tau_{\alpha}} \mathcal{F}^{\prime}\left(\frac{t}{\tau_{\alpha}}\right) \frac{\partial \ln \left(\tau_{\alpha}\right)}{\partial x} \equiv \chi_{x}(t)
$$

which is a non-monotonic function of time with a maximum for $t \approx \tau_{\alpha}$. We focus on the height of this maximum, $\chi_{4} \equiv \chi_{4}\left(t=\tau_{\alpha}\right)$, which can then be estimated from the 


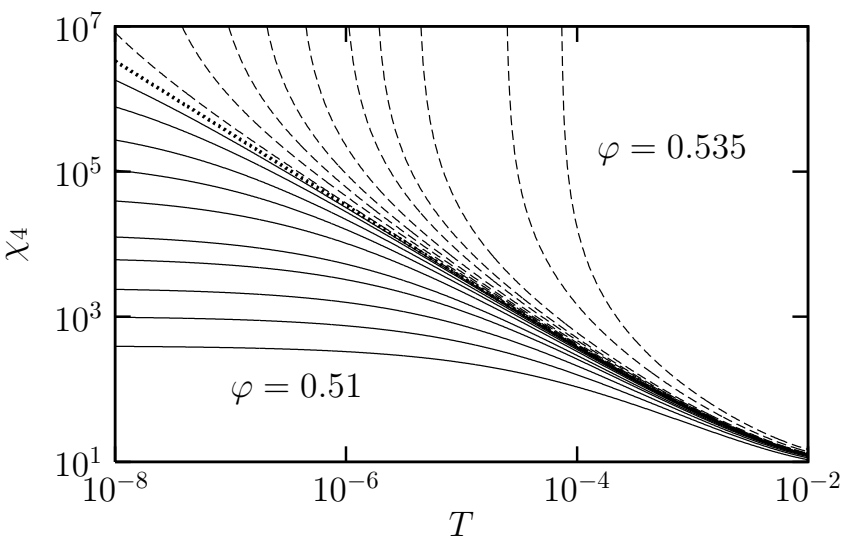

FIG. 7: Evolution of the peak of the dynamic susceptibility $\chi_{4}$ estimated from Eq. (27) for the same volume fractions of in Fig. 3 including data below (full lines), at (dotted line), and above (dashed lines) $\varphi_{c}$. The behaviour is clearly reminiscent of the one of $\tau_{\alpha}(\varphi, T)$ in Fig. 3 .

behaviour of the relaxation time alone:

$$
\chi_{4}=C^{2}\left[\frac{T^{2}}{c_{V}}\left(\frac{\partial \ln \tau_{\alpha}}{\partial T}\right)^{2}+S(0, T) \varphi^{2}\left(\frac{\partial \ln \tau_{\alpha}}{\partial \varphi}\right)^{2}\right],
$$

with $C=\mathcal{F}^{\prime}(1)$, so that $C=\beta e^{-1}$ for a stretched exponential lineshape, $\mathcal{F}(x) \sim \exp \left(-x^{\beta}\right)$.

To proceed analytically we make use of the scaling form in Eq. (14) for the relaxation time. We get:

$\chi_{4} / C^{2}=\frac{1}{4 c_{V}} G_{ \pm}^{2}(x)+S(0, T)\left(\frac{\gamma^{\mathrm{HS}} \varphi}{|\delta \varphi|}\right)^{2}\left(1-G_{ \pm}(x) / \gamma^{\mathrm{HS}}\right)^{2}$,

where $G_{ \pm}(x) \equiv x f_{ \pm}^{\prime}(x) / f_{ \pm}(x)$.

In Fig. 7 we show the evolution of $\chi_{4}$, evaluated from Eq. (27) across $\varphi_{c}$. It is clear from this figure that $\chi_{4}$ has scaling properties very similar to the ones of the relaxation time (Fig. 3). It increases and saturates to a plateau when $T$ decreases for $\varphi<\varphi_{c}$, obeys a power law behaviour at $\varphi_{c}$, and diverges algebraically at $T_{c}(\varphi)$ above $\varphi_{c}$.

In particular, we find that the term proportional to $c_{V}^{-1}$ and stemming from the temperature derivative in Eq. (24) is always safely negligible to the volume fraction derivative term in the dynamic range shown in Fig. 7. In fact, this figure would be almost unchanged if we had shown only the second term in Eq. (24). Physically this implies, not too surprisingly, that dynamic heterogeneity in the scaling regime of the harmonic sphere system is mainly controlled by density fluctuations, just as for hard spheres [2], while energy fluctuations play little role. We note that the opposite is true in supercooled liquids, where density fluctuations seem to be generically dominated by energy fluctuations [37, 39].

A second interesting consequence is that the scaling behaviour of $\chi_{4}$ near $\varphi_{c}$ can then be obtained analyti-

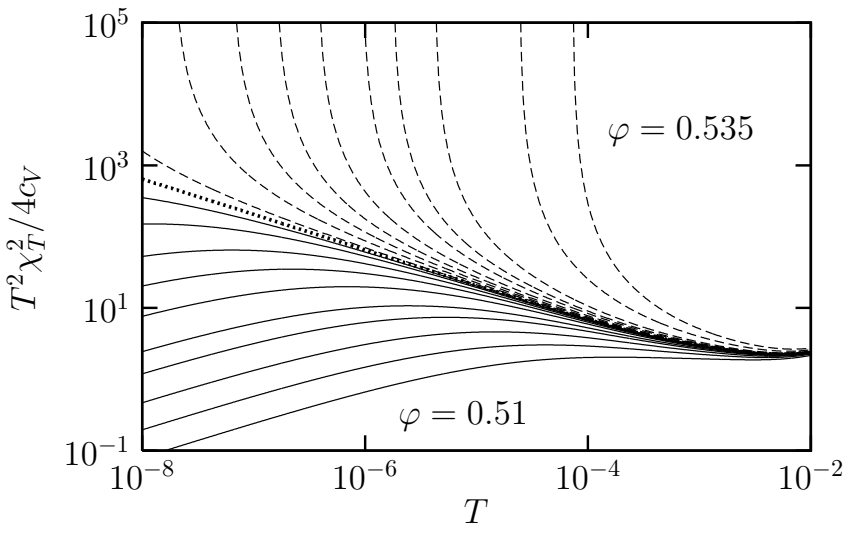

FIG. 8: Evolution of the contribution of the term containing the temperature in Eq. (24) for the same parameters and using the same representation as in Fig. 7 Note that the vertical scale in both figures is different, and that the thermal contribution to $\chi_{4}$ is always much smaller than the density contribution.

cally:

$$
\chi_{4} \approx \chi_{4}^{\mathrm{HS}}(\varphi) \mathcal{X}_{ \pm}\left(\frac{|\delta \varphi|}{\sqrt{T}}\right)
$$

where

$$
\chi_{4}^{\mathrm{HS}}(\varphi) \sim \varphi^{2} /\left(\varphi_{c}-\varphi\right)^{2},
$$

is the hard sphere result [39], and $\mathcal{X}_{ \pm}(x)=(1-$ $\left.G_{ \pm}(x) / \gamma^{\mathrm{HS}}\right)^{2}$. The scaling behaviour in Eq. (28) is similar to the one of $\tau_{\alpha}(\varphi, T)$ found in Eq. (14). In fact the similarity is even quantitative, since combining Eqs. 114, 20, 28), we can explicitly show that the relationship between the four-point susceptibility and the averaged relaxation time is identical for soft and hard spheres in the scaling regime near $\varphi_{c}$, up to subleading contributions. This suggests that plotting $\chi_{4}(\varphi, T)$ vs. $\tau_{\alpha}(\varphi, T)$ would collapse all data for harmonic spheres onto the hard sphere data.

For completeness we also show the 'thermal' contribution to $\chi_{4}(t)$ in Eq. (24) in Fig. 8, because this term is too small to have observable effects in Fig. (7) Although its shape seems similar to the one of $\chi_{4}$, it is not quite the same: it vanishes as $T \rightarrow 0$ for $\varphi<\varphi_{c}$, because $\tau_{\alpha}$ does not depend on $T$ in this limit. It follows a power law behaviour for $\varphi=\varphi_{c}$, but this divergence is in fact entirely due to the $1 / c_{V}$ prefactor, since the specific heat behaves as $c_{V}(T) \sim \sqrt{T}$ from Eq. (9). Finally, for $\varphi>\varphi_{c}$ both terms contributing to $\chi_{4}$ diverge in the same power law manner, as $\left(T-T_{c}(\varphi)\right)^{-2}$, but the respective amplitude of the two terms is set by $(\varphi / \delta \varphi)^{2}$ and $1 / c_{V}$. This implies that as long as $\varphi$ is close to $\varphi_{c}$ the density derivative term dominates over the temperature derivative term. It is only when $\varphi$ is much larger than $\varphi_{c}$ that the temperature contribution might become dominant, but $\chi_{4}$ is not described by Eq. (27) anymore and a direct evaluation of all contributions would be required to investigate this 
crossover at large volume fractions. We have not pursued these investigations.

To the best of our knowledge, dynamic heterogeneity has not been discussed numerically in harmonic spheres, and we cannot compare the present results with numerical results. However, for hard spheres, it has been established that the power law scaling in Eq. (29) is barely visible on actual data [2], and dynamic correlations seem to increase much more slowly with increasing the density than predicted by MCT, as is found also for supercooled liquids [37].

We note again, however, the close similarity between the present MCT results for dynamic correlations and the scaling properties found in driven athermal simulations of harmonic spheres where algebraic divergences of spatial correlations of particle dynamics and scaling properties very similar to Fig. 7 were reported [18, 19, 41].

\section{DISCUSSION}

In this paper, we have investigated theoretically the behaviour of dense assemblies of harmonic spheres at low temperatures in a broad range of volume fractions, encompassing the glass transition of hard spheres at $\varphi_{c}$. We have combined hyper-netted chain closure for the structure with mode-coupling theory for the dynamics. We find that for finite temperatures near $\varphi_{c}$, harmonic spheres behave effectively as hard spheres with a renormalized volume fraction. This directly implies a scaling form for the relaxation time in the part of the volume fraction - temperature phase diagram in the vicinity of the hard sphere transition, which applies also to the amplitude of dynamic heterogeneity. At larger volume fraction, deviations from hard sphere behaviour arise, and dynamic scaling breaks down.

When compared to numerical simulations of the dynamics of harmonic spheres at thermal equilibrium, the known shortcomings of mode-coupling predictions clearly show up: MCT predicts algebraic singularities that are not observed in simulations, and fails to predict the 'activated' scaling behaviour observed in simulations of both hard and soft particles. In particular, using a mode-coupling approach we cannot discuss the large change of glass fragility with volume fraction discussed in Refs. 11, 12], as these come from subtle deviations from an Arrhenius behaviour which is not predicted with MCT. Another feature which is not well captured by the present calculations is the volume fraction dependence of the MCT critical exponent which increases with $\varphi$ in the simulations, but decreases in our calculations. It is not clear, however, whether this last failure originates from the approximation used for the structure factor or from the mode-coupling theory itself.

Although MCT is qualitatively unable to describe the nature of the glass transition in harmonic spheres, there seems to exist a time window of approximately 3 decades where its predictions can be applied. In experiments with hard sphere colloids, it is in fact only very recently that deviations from MCT behaviour were unambiguously observed in experiments covering a very broad range of relaxation times 2]. This means in turn that for 'standard' experiments the behaviour predicted in the present article might still be of some value, and our theoretical approach could certainly be extended to a broader family of soft pair potentials beyond harmonic interactions. Thus, we hope that the present results will motivate further analysis of the dynamics of soft colloids in simulations and experiments.

Although we mentioned in the introduction that soft colloids are currently studied by several groups, the glass transition of soft colloids has only very recently been studied in a system made of microgel particles [7]. In this paper, three types of particles with increasing softness were studied. The most striking result of this study is a change of the volume fraction dependence of the relaxation time with softness, from $\tau_{\alpha} \sim\left(\varphi_{c}-\varphi\right)^{-\gamma^{\mathrm{HS}}}$ for hard particles, to $\log \left(\tau_{\alpha}\right) \propto \varphi$ for very soft particles. Since the interparticle interaction in this system is not known, one could imagine using a potential such as in Eq. (1), with increasing temperature playing the role of increasing particle softness. Our results in fact predict that the volume fraction dependence of the relaxation time does not vary from the hard sphere behaviour for a very broad range of temperatures of at least 6 decades, see Fig. 3. Since the qualitative behaviour found in this work should be independent of the details of the pair potential, this suggests that, very likely, the change of particle softness in Ref. 7] also corresponds to a change of the form of the interaction between particles, and is thus difficult to explain theoretically on the basis of the present work.

Does the scaling behaviour found for harmonic spheres teach some lessons for understanding the glass transition of molecular glass-formers? A major conclusion drawn from the present theoretical results is that the physics of harmonic spheres is simply the one of hard spheres: they undergo a glass transition both upon compression or upon cooling with a relaxation time which diverges when the effective volume fraction $\varphi_{\mathrm{eff}}(\varphi, T)$ of the harmonic spheres system becomes equal to $\varphi_{c}$, the hard sphere critical packing fraction. Moreover, the physics for $\varphi_{\text {eff }}(\varphi, T) \lesssim \varphi_{c}$ is the same for both soft and hard particles, as seen for instance from the behaviour of the dynamic susceptibilities discussed in Sec. V.

For this physical behaviour to be useful to understand aspects of the glass transition, one should invoke the possibility that real liquids can be effectively described as hard spheres, an assumption which has a long history in the field of liquid state theory [20]. It was revisited very recently in the present context in Ref. 42] which established that the dynamics of real liquids, and in particular the interplay between density and temperature, is qualitatively different from the one of soft particles with a finite interaction range such as harmonic spheres. For instance the large change of glass fragility observed for 
harmonic spheres is not observed in molecular liquids, which obey a much simpler scaling yielding glass fragilities independent of the density [42]. Here, we also found that the main contribution to the dynamic susceptibility in Eq. (24) is always given by the density contribution, while in real liquids the temperature term dominates [37, 39, 40], suggesting that a different physics is at play in both cases. It could be, for instance, that attractive forces not included in potentials such as Eq. (1) provide a non-negligible contribution to the energy barriers that need to be crossed during structural relaxation.

Finally, we comment on the intriguing similarity emphasized throughout this paper between the present results and the dynamic scaling behaviour discussed in several recent articles dealing with the athermal, driven dynamics of harmonic spheres [18, 19, 41]. In both cases, algebraic divergences of dynamical quantities are obtained in the hard sphere limit, with a dynamic scaling behaviour observed in its vicinity both at the level of the averaged dynamics and of the dynamical fluctuations. Note, however, the very different nature of the critical density in both cases [30]: the hard sphere glass transition discussed here is defined from the divergence of an equilibrium quantity, which is thus by definition independent of the preparation protocol of the system. Instead the zero temperature jamming transition does explicitly depend on which ensemble of configurations is selected by the studied dynamics [16, 31, 43, 44]. There is no limit where these two distinct transitions can merge.

We believe that the deep underlying explanation of this similarity is the fact that in both cases, the dynamics is controlled by the existence of 'soft modes', meaning that relaxation proceeds both within MCT and in athermal dynamics without spontaneous crossing of energy barriers. In both cases, thus, nontrivial collective dynamics stems from the existence of nearly flat directions of the potential energy landscape [30, 41]. This is a clear shortcoming of MCT when it deals with the glass transition of glass-forming liquids, but since no such barrier crossing takes place in the $T=0$ driven dynamics relevant for granular systems, we suggest that 'mean-field' modecoupling approaches such as the ones developed here and by others [31] provide good starting points to describe the dynamics of soft particles near the jamming transition at zero temperature.

\section{Acknowledgments}

We thank L. Cipelletti, W. Kob, G. Tarjus, T. Witten, F. Zamponi for useful exchanges about this work. This work was started when G. Szamel was on sabbatical leave at LCVN. He is grateful to his colleagues there for their hospitality and to CNRS for financial support that made his stay in Montpellier possible. G. Szamel and E. Flenner gratefully acknowledge the support of NSF Grant No. CHE 0517709. H. Jacquin acknowledges financial support from Capital Fund Management (CFM) Foundation, and from the LCVN in the early stages of this work. L. Berthier is partially funded by ANR Dynhet.
[1] P. N. Pusey and W. van Megen, Nature 320, 340 (1986).

[2] G. Brambilla, D. El Masri, M. Pierno, G. Petekidis, A. B. Schofield, L. Berthier, and L. Cipelletti, Phys. Rev. Lett. 102, 085703 (2009).

[3] J. D. Bernal and J. Mason, Nature 188, 910 (1960).

[4] Z. Zhang, N. Xu, D. T. N. Chen, P. Yunker, A. M. Alsayed, K. B. Aptowicz, P. Habdas, A. J. Liu, S. R. Nagel, and A. G. Yodh, Nature 459, 230 (2009).

[5] D. A. Sessoms, I. Bischofberger, L. Cipelletti, and V. Trappe, Phil. Trans. R. Soc. A 367, 5013 (2009).

[6] S. Mazoyer, F. Ebert, G. Maret, and P. Keim, arXiv:0903.4675

[7] J. Mattsson, H. M. Wyss, A. Fernandez-Nieves, K. Miyazaki, Z. Hu, D. R. Reichman, D. A. Weitz, Nature 462 (7269), 83 (2009).

[8] S. Mazoyer, L. Cipelletti, and A. Duri, Nat. Phys. 4, 550 (2008).

[9] J. Goyon, A. Colin, G. Ovarlez, A. Ajdari, and L. Bocquet, Nature 454, 7200 (2008).

[10] X. Cheng, arXiv:0905.2788 (2009).

[11] L. Berthier and T. A. Witten, EPL 86, 10001 (2009).

[12] L. Berthier and T. A. Witten, Phys. Rev. E 80021502 (2009).

[13] N. Xu, T. K. Haxton, A. J. Liu, and S. R. Nagel, arXiv:0910.0666
[14] N. Xu, arXiv:0911.1576

[15] D. J. Durian, Phys. Rev. Lett. 75, 4780 (1995).

[16] C. S. O'Hern, S. A. Langer, A. J. Liu, and S. R. Nagel, Phys. Rev. Lett. 88075507 (2002).

[17] C. Heussinger and J.-L. Barrat, Phys. Rev. Lett. 102, 218303 (2009).

[18] P. Olsson and S. Teitel, Phys. Rev. Lett. 99, 178001 (2007).

[19] T. Hatano, J. Phys. Soc. Jpn. 77, 123002 (2008).

[20] J. P. Hansen and I. R. McDonald, Theory of Simple Liquids (Elsevier, Amsterdam, 1986).

[21] W. Götze, Complex dynamics of glass-forming liquids: A mode-coupling theory (Oxford University Press, Oxford, 2008).

[22] J. C. Pamies, A. Cacciuto, and D. Frenkel, J. Chem. Phys. 131, 044514 (2009).

[23] F. H. Stillinger, J. Chem. Phys. 65, 3968 (1976).

[24] A. A. Louis, P. G. Bolhuis, and J. P. Hansen, Phys. Rev. E 62, 7961 (2000).

[25] U. Bengtzelius, W. Götze, and A. Sjölander, J. Phys. C: Solid State Phys. 17, 5915 (1984).

[26] G. Szamel and H. Löwen, Phys. Rev. A 44, 8215 (1991).

[27] M. Fuchs, W. Götze, I. Hofacker, and A. Latz, J. Phys.: Condens. Matter 3, 5047 (1991).

[28] E. Flenner and G. Szamel, Phys. Rev. E 72, 031508 
(2005).

[29] G. Brambilla, D. El Masri, M. Pierno, G. Petekidis, A. B. Schofield, L. Berthier, and L. Cipelletti, J. Stat. Mech. P07015 (2009).

[30] F. Krzakala and J. Kurchan, Phys. Rev. E 76, 021122 (2007).

[31] R. Mari, F. Krzakala and J. Kurchan, Phys. Rev. Lett. 103, 025701 (2009).

[32] G. Biroli and J.-P. Bouchaud, Europhys. Lett. 67, 21 (2004).

[33] M. D. Ediger, Annu. Rev. Phys. Chem. 51, 99 (2000).

[34] S. Franz and G. Parisi, J. Phys.: Condens. Matter 12, 6335 (2000).

[35] C. Toninelli, M. Wyart, L. Berthier, G. Biroli, and J.-P. Bouchaud, Phys. Rev. E 71, 041505 (2005).

[36] P. Mayer, H. Bissig, L. Berthier, L. Cipelletti, J. P. Garrahan, P. Sollich, and V. Trappe, Phys. Rev. Lett. 93, 115701 (2004).

[37] C. Dalle-Ferrier, C. Thibierge, C. Alba-Simionesco, L.
Berthier, G. Biroli, J.-P. Bouchaud, F. Ladieu, D. L'Hôte, and G. Tarjus, Phys. Rev. E 76, 041510 (2007).

[38] L. Berthier, G. Biroli, J. P. Bouchaud, L. Cipelletti, D. El Masri, D. L'Hote, F. Ladieu, and M. Pierno, Science 310, 1797 (2005).

[39] L. Berthier, G. Biroli, J.-P. Bouchaud, W. Kob, K. Miyazaki, and D. R. Reichman, J. Chem. Phys. 126, 184503 (2007).

[40] L. Berthier, G. Biroli, J.-P. Bouchaud, W. Kob, K. Miyazaki, and D. R. Reichman, J. Chem. Phys. 126, 184504 (2007).

[41] T. Hatano, Phys. Rev. E 79, 050301(R) (2009).

[42] L. Berthier and G. Tarjus, Phys. Rev. Lett. 103, 170601 (2009).

[43] A. Donev, S. Torquato, F. H. Stillinger, and R. Connelly, Phys. Rev. E 70, 043301 (2004).

[44] P. Chaudhuri, L. Berthier, and S. Sastry, arXiv:0910.0364 